Article

\title{
Experimental Observation of Temperature-Driven Topological Phase Transition in HgTe/CdHgTe Quantum Wells
}

\author{
Maksim S. Zholudev ${ }^{1, *}$, Aleksandr M. Kadykov ${ }^{1,2}{ }^{(0)}$, Mikhail A. Fadeev ${ }^{1,2}$, \\ Michal Marcinkiewicz ${ }^{2}$, Sandra Ruffenach ${ }^{2}$, Christophe Consejo ${ }^{2}$, Wojciech Knap ${ }^{2,3}$, \\ Jeremie Torres ${ }^{4}$, Sergey V. Morozov ${ }^{1}$, Vladimir I. Gavrilenko ${ }^{1}$, Nikolay N. Mikhailov ${ }^{5}$, \\ Sergey A. Dvoretskii ${ }^{5}$ (D) and Frederic Teppe ${ }^{2}$ \\ 1 Department for physics of semiconductors, Institute for Physics of Microstructures RAS, GSP-105, \\ 603950 Nizhny Novgorod, Russia; alexander.kadykov@gmail.com (A.M.K.); fadeev@ipmras.ru (M.A.F.); \\ more@ipmras.ru (S.V.M.); gavr@ipmras.ru (V.I.G.) \\ 2 Laboratoire Charles Coulomb, UMR CNRS 5221, University of Montpellier, Place Eugène Bataillon-CC069, \\ 34095 Montpellier, France; michal.marcinkiewicz@umontpellier.fr (M.M.); \\ Sandra.Ruffenach@umontpellier.fr (S.R.); Christophe.Consejo@umontpellier.fr (C.C.); \\ wojciech.knap@umontpellier.fr (W.K.); Frederic.Teppe@umontpellier.fr (F.T.) \\ 3 Center for Terahertz Research and Applications (CENTERA), Institute for High Pressure Physics, \\ Polish Academy of Sciences, 29/37 Sokołowska Str, 01-142 Warsaw, Poland \\ 4 Institut d'Electronique et des Systemes, University of Montpellier, 860, rue Saint Priest, Bâtiment 5-CC 05001, \\ 34090 Montpellier, France; jeremie.torres@umontpellier.fr \\ 5 Institute of Semiconductor Physics, SB RAS, 630090 Novosibirsk, Russia; mikhailov@isp.nsc.ru (N.N.M.); \\ dvor@isp.nsc.ru (S.A.D.) \\ * Correspondence: zholudev@ipmras.ru
}

Received: 28 January 2019; Accepted: 28 February 2019; Published: 1 March 2019

\begin{abstract}
We report on the comparison between temperature-dependent magneto-absorption and magnetotransport spectroscopy of $\mathrm{HgTe} / \mathrm{CdHgTe}$ quantum wells in terms of the detection of the phase transition between the topological insulator and band insulator states. Our results demonstrate that temperature-dependent magnetospectroscopy is a powerful tool to discriminate trivial and topological insulator phases, yet the magnetotransport method is shown to have advantages for the clear manifestation of the phase transition with accurate quantitative values of the transition parameter (i.e., critical magnetic field $B_{c}$ ).
\end{abstract}

Keywords: topological insulator; phase transition; mercury cadmium telluride; Landau levels; magneto-absorption; magnetotransport

\section{Introduction}

Due to their amazing electronic properties, 2D and 3D topological insulators (TI) have been attracting increasing attention during the last decade. The HgTe/CdHgTe quantum well (QW) was the first system where the TI state was predicted [1] and experimentally observed [2]. The inversion of the electron-like level $\mathrm{E} 1$ and the hole-like level H1 induces spin-polarized helical edge states [2]. When E1 and H1 levels cross each other, the band structure mimics a linear dispersion of massless Dirac fermions [3] corresponding to the phase transition between the trivial band insulator (BI) state and the TI state.

By fabricating QWs with different thicknesses, the band structure can be widely tuned. For $\mathrm{HgTe} / \mathrm{Cd}_{0.7} \mathrm{Hg}_{0.3} \mathrm{Te} \mathrm{QW}$ grown on CdTe buffer the band structure inverted if QW thickness $d$ exceeds the critical value $d_{c}=6.3 \mathrm{~nm}$, whereas for $d<d_{c}$ the band structure is normal [1]. At the critical thickness, the band gap closes, establishing single-valley 2D massless Dirac fermions [3]. 
As well as the QW thickness, temperature [4,5] and hydrostatic pressure [6] also induce the transition between the BI and TI phases via the critical gapless state. The temperature effect on the band ordering in $\mathrm{HgTe} / \mathrm{CdHgTe}$ QWs is mainly caused by a strong temperature dependence of the energy gap at the $\Gamma$ point of the Brillouin zone between the $\Gamma 6$ and $\Gamma 8$ bands in $\mathrm{HgCdTe}$ crystals [7]. In essence, temperature changes (decreases) the critical thickness of $\mathrm{HgTe} / \mathrm{CdHgTe} Q W s$. The point where the critical thickness is equal to the actual size of the well is the critical temperature $T_{\mathcal{C}}$.

Any phase transition implies a discontinuous change of some parameter, e.g., specific volume (melting, boiling) or the first derivative of the order parameter (ferromagnetic, superconductors). The order parameter corresponding to the edge states of the TI phase can be introduced in theory but it is hardly useful in practice because the detection of these states is very difficult. Fortunately, there are other parameters changing abruptly between the TI and BI phases that can be experimentally measured. The first one is the dependence of the energy gap on temperature, which has a negative derivative below the critical point and a positive derivative above it. The main flaw of the direct band gap measurements is that measuring a small energy gap by light absorption or transmission is very difficult even at liquid helium temperature and almost impossible at higher temperatures.

Another possible approach is based on the analysis of the behavior of Landau levels (LLs) in high magnetic fields. Figure 1 illustrates a typical LL configuration for BI, zero-gap and TI cases with the transitions that are usually observed in magnetoabsorption experiments. One of the inherent properties of the inverted band $\mathrm{HgTe} / \mathrm{CdHgTe} \mathrm{QWs}$ is the behavior of zero-mode LLs with indices $\mathrm{N}$ $=0$ and $N=-2$ (bold lines on Figure 1). In the BI phase these levels belong to conduction and valence bands respectively and do not cross (Figure 1a). In the TI phase (Figure 1c), zero-mode LLs have reverse start positions (at $B=0$ ) and consequently cross at a critical magnetic field $B_{c}$, above which an inverted band ordering is transformed into a normal one [2]. The latter can be conditionally interpreted as negative values for $B_{c}$. Thus, $B_{c}=0$ corresponds to a topological phase transition between the BI and TI phases. As well as the band ordering, a critical magnetic field also depends on temperature and pressure, and therefore, can be varied by tuning these external parameters [6].
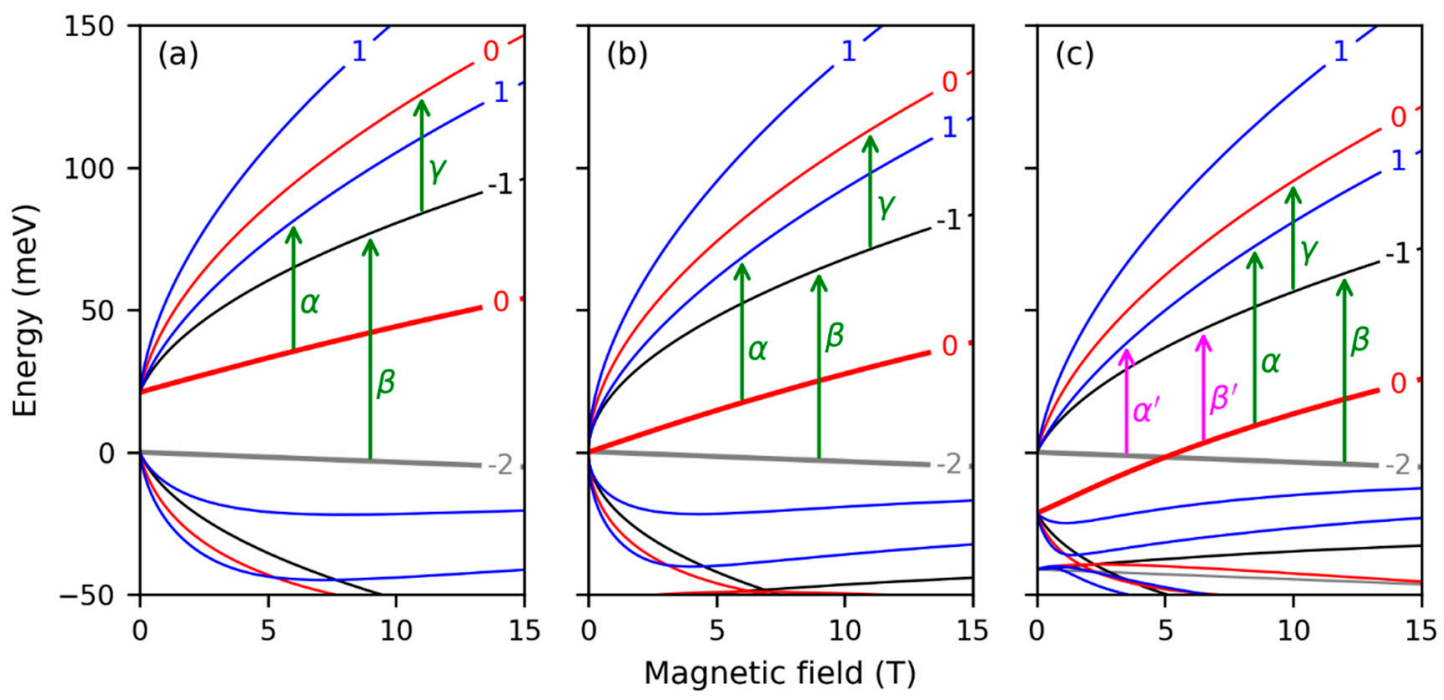

Figure 1. Landau levels of $\mathrm{HgTe} / \mathrm{Cd}_{0.7} \mathrm{Hg}_{0.3} \mathrm{Te} \mathrm{QW}$ calculated within axial approximation for: (a) band insulator $(d=5.5 \mathrm{~nm})$; $(\mathbf{b})$ zero-gap semiconductor $(d=6.3 \mathrm{~nm})$; $(\mathbf{c})$ topological insulator $(d=7.4 \mathrm{~nm})$. Zero-mode LLs are shown with bold lines. LL indices are shown with both numbers on the lines and line colors. We use the same calculation method and LL indices notation of as in [8,9].

By analyzing the magnetotransport data at high and low temperatures Wiedmann et al. [5] have experimentally shown that the TI phase vanishes with temperature. However, due to high critical temperature $T_{c}$ (above $200 \mathrm{~K}$ ) the gapless state could not be directly observed in the samples studied. Ikonnikov et al. [10] have reported on magnetospectroscopy of HgTe quantum wells in 
magnetic fields up to $45 \mathrm{~T}$ in the temperature range from $4.2 \mathrm{~K}$ to $185 \mathrm{~K}$. They show that although their samples are TI only at low temperatures, the signature of the TI phase persists in optical transitions at high temperatures and high magnetic fields. In our previous works we have reported on the observation of temperature driven topological phase transition using far-infrared magnetoabsorption spectroscopy [11] and magnetotransport [12,13]. In the present work we analyze these two methods to make a conclusion about their applicability for the TI-BI phase transition investigation.

\section{Results}

It is clear from Figure 1a that in BI state $\alpha$ is an intraband transition within the conduction band and $\beta$ is an interband transition. In TI state (Figure 1c) $\alpha$ is an interband transition and $\beta$ is an intraband transition. Both $\alpha$ and $\beta$ lines have been observed in our experiment [11] (see Figure 2). The position of interband line should give us the band gap value when followed down to the zero magnetic field. Unfortunately, doing this was impossible in the framework of our experiments [11]. Instead of that in the work [11], we had to use extrapolation of experimentally observed $\alpha$ and $\beta$ transition lines to estimate the band gap. This approach, however, is not reliable because of the specific type of functions used for extrapolation (Figure 3). For these square-root-like functions a small difference at high magnetic fields results in a significant difference at the zero magnetic field. Hence, a small error in the experimental data leads to a big error in the estimated energy gap. This effect is especially strong in inverted-band QWs because of the disagreement of theoretical and experimental line positions (see Figure $2 \mathrm{f}, \mathrm{g}$ ). Consequently, we should conclude that we cannot trust the energy gap values reported in [11].

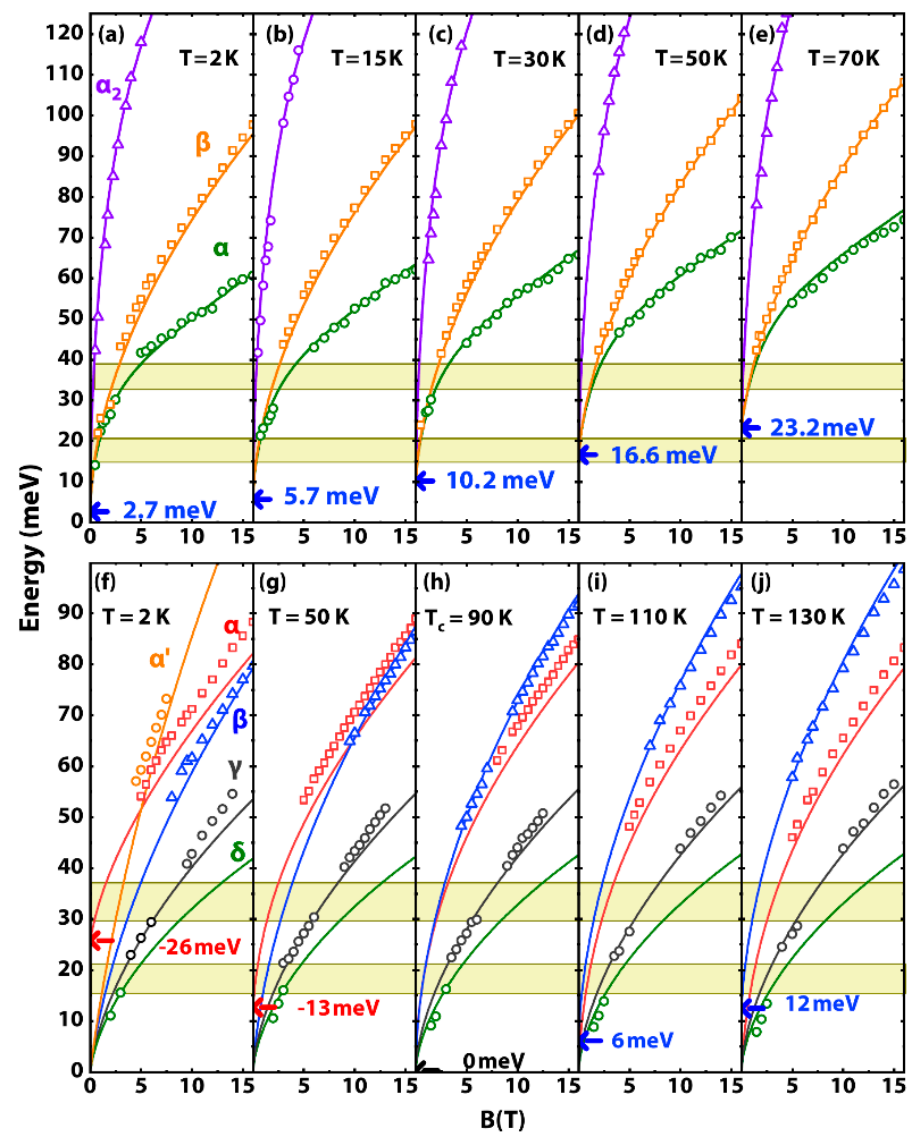

Figure 2. Fan chart of inter-LL transitions in 6-nm wide $\mathrm{HgTe} / \mathrm{Cd}_{0.62} \mathrm{Hg}_{0.38} \mathrm{Te} \mathrm{QW}(\mathbf{a}-\mathbf{e})$ and 8-nm wide width $\mathrm{HgTe} / \mathrm{Cd}_{0.8} \mathrm{Hg}_{0.2} \mathrm{Te} \mathrm{QW}(\mathbf{f}-\mathbf{j})$ from work [11]. The calculated transitions are shown in solid lines while the experimental data are represented by the open symbols in the same colors as the theoretical curves. The estimated values of the energy gap are shown by the horizontal arrows. Shaded areas indicate the $\mathrm{GaAs}$ and $\mathrm{HgCdTe}$ reststrahlen bands. 


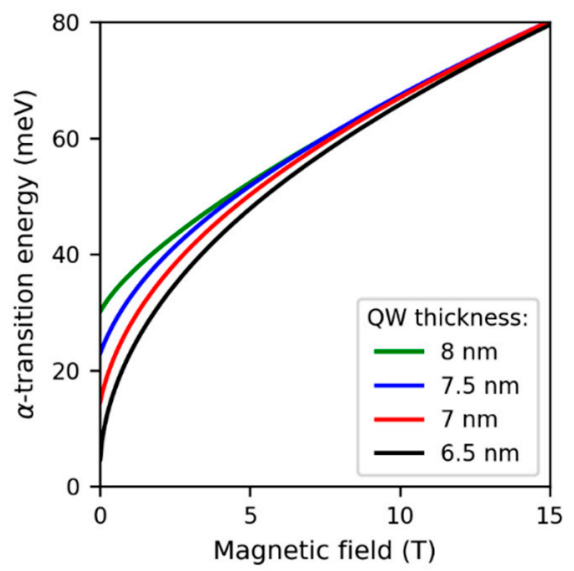

Figure 3. Calculated energy of $\alpha$-transition for $\mathrm{HgTe} / \mathrm{Cd}_{0.7} \mathrm{Hg}_{0.3} \mathrm{Te} \mathrm{QWs}$ of different thickness.

The fact of the phase transition, however, can still be observed from these experimental data. To do this we suggest another approach based on the qualitative difference in the structure of the transition lines in TI and BI. For TI state there are two transitions, $\alpha^{\prime}$ and $\beta^{\prime}$ (see Figure 1c) that are forbidden by the selection rules in the simple model we use. For electric-dipole excitations in Faraday geometry a transition can change LL number $N$ by \pm 1 and cannot change its spin projection on the $\mathrm{z}$ axis. Transition $\alpha^{\prime}$ is not allowed because it changes the LL number by 3 and $\beta^{\prime}$ implies a spin flip (for more details see e.g., [14]). The corresponding lines, however, can be observed because of mixing of the zero-mode LLs near the crossing point $[8,15]$ (line $\alpha^{\prime}$ is shown on Figure 2f). Hence in magnetoabsorption experiments we cannot observe the crossing of LLs directly, but we can see the consequences of LL mixing (i.e., emergence of lines $\alpha^{\prime}$ and $\beta^{\prime}$ ) allowing detection of the TI state. The limitation of this method is that anticrossing can be clearly seen only when $B_{c}$ is high enough so that $\alpha$ and $\beta$ transitions are not forbidden by the Pauli principle and the crossing point does not fall in the restrahlen bands (see Figure 2f). Consequently, in the vicinity of the conditions corresponding to the phase transition when $B_{c}$ is close to zero no manifestation of the LL anticrossing can be observed (see Figure 2g,h).

Another approach is to analyze $\alpha$ and $\beta$ lines in high magnetic fields where they are clearly seen at all temperatures. In Figure 1 one can see that the energy of the lower level of $\alpha$ transition $(N=0)$ goes up with the magnetic field while the energy of the lower level of $\beta$ transition $(N=-2)$ goes down. On the other hand, the energy of the upper levels $(N=1$ and $N=-1)$ goes up for both transitions. Thus, the energy of $\beta$ line will always have a higher derivative over the magnetic field than the one of $\alpha$ line. Because of this in BI state $\beta$ line lies above $\alpha$ line and never crosses it (Figure 2a-e). Our calculations show that in TI state $\beta$ line starts from zero and should cross $\alpha$ line at some point, however, in the experiment we see two parallel lines (see e.g., Figure 2f,g). We believe that it comes from the magnetic field being too weak to reach the $\alpha-\beta$ crossing point. The lines look parallel because their energies close to $B_{c}$ are affected by the aforementioned $\alpha-\alpha^{\prime}$ and $\beta-\beta^{\prime}$ anticrossing $[8,15]$. Hence at these conditions the behavior of the $\alpha$ and $\beta$ lines predicted by a simple axial model cannot be observed due to interference with low symmetry effects around $B_{c}$ and the limitation of the maximal magnetic field. At critical temperature the crossing of $\alpha$ and $\beta$ lines was observed (Figure 2h). Above the critical temperature we can clearly see that $\beta$ line (that has higher slope) lies above $\alpha$ line as expected for BI state (Figure 2i,j).

The alternative method that allows detection of the topological phase transition is based on magnetotransport measurements in a gated Hall bar [13]. The ability to set the Fermi level in a wide range by the applied gate voltage $\mathrm{V}_{\mathrm{g}}$, allows one to perform a magnetotransport mapping of the structure of LLs of the sample. As it was proposed in the pioneer paper by Büttner et al. [3], the peaks of $\partial \sigma_{x y} / \partial V_{g}$ curves give the precise positions of crossings between the Fermi level and 
the LLs. Therefore plotting $\partial \sigma_{x y} / \partial V_{g}$ for each magnetic field value makes it possible to reveal the dispersion of the LLs (see Figure 4).

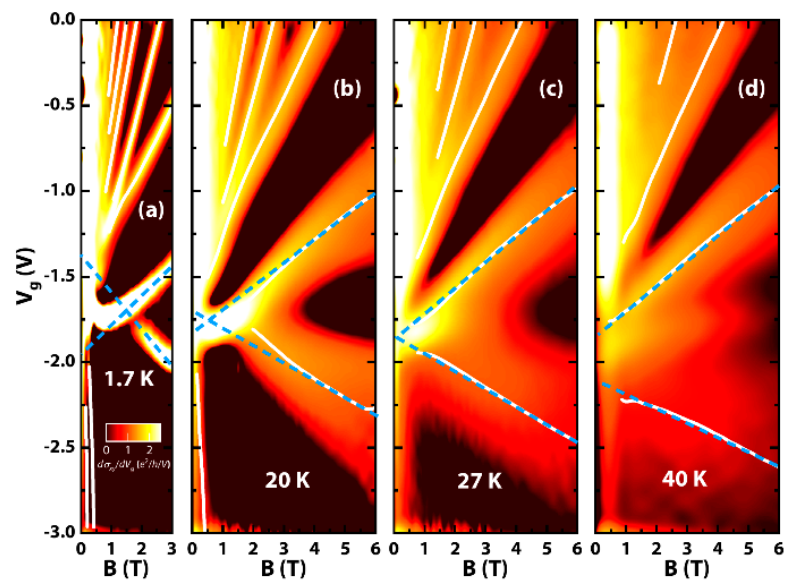

Figure 4. Colormap of the experimental Landau level fan charts of derivative $\partial \sigma_{x y} / \partial V_{g}$ in 6.5-nm wide $\mathrm{HgTe} / \mathrm{Cd}_{0.65} \mathrm{Hg}_{0.35} \mathrm{Te} \mathrm{QW}$ as a function of both magnetic field and gate voltage from work [13]; brighter color represents higher value of the derivative; dark color represents a lower value. The white curves present positions of $\sigma_{x y}=(2 n+1) e^{2} /(2 h)$. The blue dashed curves fit the experimental behavior of the zero-mode LLs based on $\sigma_{x y}$ values at high magnetic fields.

In the work [13] we define the critical magnetic field $B_{c}$ as the position of the crossing of zero-mode LLs or their linear extrapolation. Consequently, the value of $B_{c}$ can be negative in the normal semiconductor phase. It is necessary to note that the extrapolation we use is much more accurate than the one used in magnetooptical experiments. The measurements have shown that when the Fermi level is located within the conduction or the valence band the value of the 2D carrier concentration depends linearly on the gate voltage $V_{g}$ [16]. Consequently, as far as the LL degeneracy rate is also proportional to the 2D carrier concentration, all LLs in Figure 4 are exactly linear everywhere except the range where the Fermi level lies in the gap $\left(-2 \mathrm{~V}<V_{g}<-1.5 \mathrm{~V}\right)$. Thus, the method used in work [13] allows precise measurement of both positive and negative $B_{c}$ values (see Figure 5) and is a reliable instrument to probe a temperature-induced phase transition between the BI and TI phases.

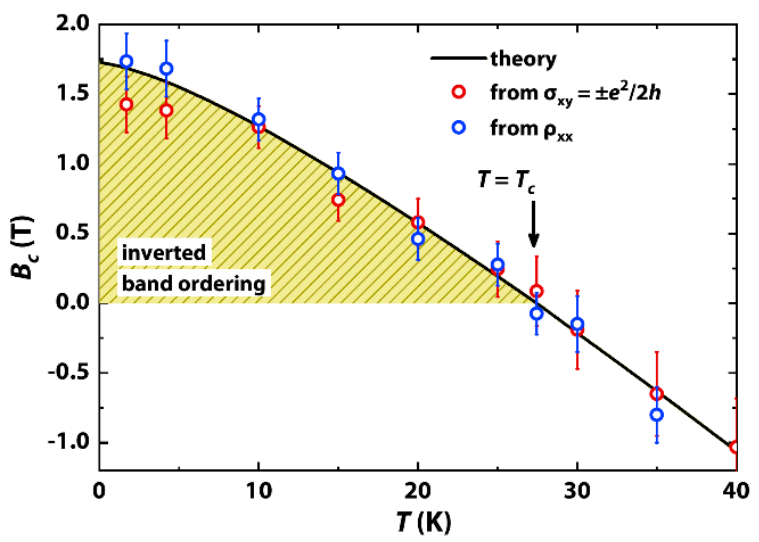

Figure 5. Theoretical (black curve) and experimental (open symbols) values for critical magnetic field as a function of temperature for $6.5 \mathrm{~nm} \mathrm{HgTe} / \mathrm{Cd}_{0.65} \mathrm{Hg}_{0.35} \mathrm{Te} \mathrm{QW}$ from work [13]. 


\section{Conclusions}

Magnetotransport experiments give a clear manifestation of the T-driven TI-BI phase transition with accurate quantitative values of the transition parameter (i.e., critical magnetic field $B_{c}$ ). Magnetooptical experiments give only a qualitative picture and any estimations of the energy gap based on these measurements are inaccurate.

Author Contributions: Conceptualization, F.T. and S.V.M.; Data curation, A.M.K. and S.R.; Funding acquisition, F.T., W.K. and V.I.G.; Investigation, M.S.Z., A.M.K., M.A.F., M.M. and S.R.; Methodology, C.C., J.T. and F.T.; Project administration, S.V.M. and F.T.; Resources, C.C., J.T., N.N.M. and S.A.D; Software, M.S.Z.; Supervision, W.K., F.T. and V.I.G.; Validation, S.V.M.; Writing—original draft, M.S.Z. and A.M.K.; Writing-review \& editing, M.A.F and F.T.

Funding: The work was supported by the Russian Foundation for Basic Research (grants 18-32-00628 and 18-52-16009) and the Russian Ministry of Education and Science of Russian Federation (grant SP-5051.2018.5). From the French side this work was supported by MIPS department of Montpellier University through the "Occitanie Térahertz Platform", by the Languedoc-Roussillon region via the "Gepeto Terahertz platform", the ARPE project "Terasens", and the REPERE project "CATS", and by the CNRS through LIA "TeraMIR". It was also supported by Foundation for Polish Science (grant MAB/2018/9) from the funds of the European Regional Development Fund under the Smart Growth Operational Programme (SG OP).

Conflicts of Interest: The authors declare no conflict of interest. The funders had no role in the design of the study; in the collection, analyses, or interpretation of data; in the writing of the manuscript, or in the decision to publish the results.

\section{References}

1. Bernevig, B.A.; Hughes, T.L.; Zhang, S.-C. Quantum spin Hall effect and topological phase transition in HgTe quantum wells. Science 2006, 314, 1757-1761. [CrossRef] [PubMed]

2. Konig, M.; Wiedmann, S.; Brune, C.; Roth, A.; Buhmann, H.; Molenkamp, L.W.; Qi, X.-L.; Zhang, S.-C. Quantum spin Hall insulator state in HgTe quantum wells. Science 2007, 318, 766-770. [CrossRef] [PubMed]

3. Büttner, B.; Liu, C.X.; Tkachov, G.; Novik, E.G.; Brüne, C.; Buhmann, H.; Hankiewicz, E.M.; Recher, P.; Trauzettel, B.; Zhang, S.C.; et al. Single valley Dirac fermions in zero-gap HgTe quantum wells. Nat. Phys. 2011, 7, 418-422. [CrossRef]

4. Sengupta, P.; Kubis, T.; Tan, Y.; Povolotskyi, M.; Klimeck, G. Design principles for HgTe based topological insulator devices. J. Appl. Phys. 2013, 114, 043702. [CrossRef]

5. Wiedmann, S.; Jost, A.; Thienel, C.; Brüne, C.; Leubner, P.; Buhmann, H.; Molenkamp, L.W.; Maan, J.C.; Zeitler, U. Temperature-driven transition from a semiconductor to a topological insulator. Phys. Rev. B 2015, 91, 205311. [CrossRef]

6. Krishtopenko, S.S.; Yahniuk, I.; But, D.B.; Gavrilenko, V.I.; Knap, W.M.; Teppe, F. Pressure- and temperature-driven phase transitions in HgTe quantum wells. Phys. Rev. B 2016, 94, 245402. [CrossRef]

7. Teppe, F.; Marcinkiewicz, M.; Krishtopenko, S.S.; Ruffenach, S.; Consejo, C.; Kadykov, A.M.; Desrat, W.; But, D.; Knap, W.M.; Ludwig, J.; et al. Temperature-driven massless Kane fermions in HgCdTe crystals. Nat. Commun. 2016, 7, 12576. [CrossRef] [PubMed]

8. Orlita, M.; Masztalerz, K.; Faugeras, C.; Potemski, M.; Novik, E.G.; Brüne, C.; Buhmann, H.; Molenkamp, L.W. Fine structure of zero-mode Landau levels in $\mathrm{HgTe} / \mathrm{Hg}_{\mathrm{x}} \mathrm{Cd}_{1-\mathrm{x}} \mathrm{Te}$ quantum wells. Phys. Rev. B 2011, 83, 115307. [CrossRef]

9. Zholudev, M.S.; Teppe, F.; Orlita, M.; Consejo, C.; Torres, J.; Dyakonova, N.; Czapkiewicz, M.; Wróbel, J.; Grabecki, G.; Mikhailov, N.N.; et al. Magnetospectroscopy of two-dimensional HgTe-based topological insulators around the critical thickness. Phys. Rev. B 2012, 86, 205420. [CrossRef]

10. Ikonnikov, A.V.; Krishtopenko, S.S.; Drachenko, O.; Goiran, M.; Zholudev, M.S.; Platonov, V.V.; Kudasov, Y.B.; Korshunov, A.S.; Maslov, D.A.; Makarov, I.V.; et al. Temperature-dependent magnetospectroscopy of HgTe quantum wells. Phys. Rev. B 2016, 94, 155421. [CrossRef]

11. Marcinkiewicz, M.; Ruffenach, S.; Krishtopenko, S.S.; Kadykov, A.M.; Consejo, C.; But, D.B.; Desrat, W.; Knap, W.M.; Torres, J.; Ikonnikov, A.V.; et al. Temperature-driven single-valley Dirac fermions in HgTe quantum wells. Phys. Rev. B 2017, 96, 035405. [CrossRef]

12. Kadykov, A.M.; Torres, J.; Krishtopenko, S.S.; Consejo, C.; Ruffenach, S.; Marcinkiewicz, M.; But, D.; Knap, W.M.; Morozov, S.V.; Gavrilenko, V.I.; et al. Terahertz imaging of Landau levels in HgTe-based topological insulators. Appl. Phys. Lett. 2016, 108, 262102. [CrossRef] 
13. Kadykov, A.M.; Krishtopenko, S.S.; Jouault, B.; Desrat, W.; Knap, W.M.; Ruffenach, S.; Consejo, C.; Torres, J.; Morozov, S.V.; Mikhailov, N.N.; et al. Temperature-Induced Topological Phase Transition in HgTe Quantum Wells. Phys. Rev. Lett. 2018, 120, 086401. [CrossRef] [PubMed]

14. Bovkun, L.S.; Ikonnikov, A.V.; Aleshkin, V.Y.; Spirin, K.E.; Gavrilenko, V.I.; Mikhailov, N.N.; Dvoretskii, S.A.; Teppe, F.; Piot, B.A.; Potemski, M.; et al. Landau level spectroscopy of valence bands in HgTe quantum wells: Effects of symmetry lowering. J. Phys. Condens. Matter 2019, 31, 145501. [CrossRef]

15. Zholudev, M.S.; Teppe, F.; Morozov, S.V.; Orlita, M.; Consejo, C.; Ruffenach, S.; Knap, W.M.; Gavrilenko, V.I.; Dvoretskii, S.A.; Mikhailov, N.N. Anticrossing of Landau levels in $\mathrm{HgTe} / \mathrm{CdHgTe}$ (013) quantum wells with an inverted band structure. JETP Lett. 2015, 100, 790-794. [CrossRef]

16. Supplemental Material of [13]. Available online: http://link.aps.org/supplemental/10.1103/PhysRevLett. 120.086401 (accessed on 28 January 2019).

2019 by the authors. Licensee MDPI, Basel, Switzerland. This article is an open access article distributed under the terms and conditions of the Creative Commons Attribution (CC BY) license (http://creativecommons.org/licenses/by/4.0/). 